\title{
Controlling dynamics in diatomic systems
}

\author{
PRAVEEN KUMAR $^{1}$ and HARJINDER SINGH ${ }^{2 * *}$ \\ ${ }^{1}$ Department of Chemistry, Panjab University, Chandigarh 160014 \\ ${ }^{2}$ Center for Computational Natural Sciences and Bioinformatics, \\ International Institute of Information Technology, Hyderabad 500032 \\ e-mail: laltu@iiit.ac.in
}

MS received 2 May 2007; accepted 31 May 2007

\begin{abstract}
Controlling molecular energetics using laser pulses is exemplified for nuclear motion in two different diatomic systems. The problem of finding the optimized field for maximizing a desired quantum dynamical target is formulated using an iterative method. The method is applied for two diatomic systems, $\mathrm{HF}$ and $\mathrm{OH}$. The power spectra of the fields and evolution of populations of different vibrational states during transitions are obtained.
\end{abstract}

Keywords. Iterative method; optimal control theory; diatomic systems; quantum control.

\section{Introduction}

The control of chemical dynamical systems using coherent beam of light sources has been an active area of research for more than two decades. Much theoretical progress has been made by designing laser pulse shapes to produce a prescribed physical system. ${ }^{1,2}$ It is now understood that quantum mechanical interference properties are fundamental to processes occurring at the atomic and molecular level. Among the several approaches used, efforts employed using optimal control theory (OCT) ${ }^{3-8}$ to design the pulse shapes have met with considerable theoretical and experimental success for small molecular systems. These include control over branching processes such as dissociation vs. ionization in $\mathrm{HCl}, \mathrm{HF}, \mathrm{HI}, \mathrm{CO}$, and ICN demonstrated by several groups, ${ }^{9-12}$ laser control of selective bond breaking in the molecule HOD with products $\mathrm{HO}$ and D or $\mathrm{H}$ and OD. ${ }^{13-15} \mathrm{~A}$ different approach using interference between competing paths developed by Shapiro et al is exemplified by the experimental demonstration of the control of branching ratio of $\mathrm{Na}_{2}$ dissociating into sodium atoms in different electronic states. ${ }^{16-18}$ Inverse quantum mechanical control to obtain appropriate field for tracking dynamics was also explored. ${ }^{19,20}$ Artificial intelligence and learning algorithm based experimental set-ups $^{21-25}$ are also capable of providing a suitable laser field for prescribed dynamics, which prepare

*For correspondence reactants in specific states to follow a desired reaction path. This control technique is difficult to implement for complex molecules.

The OCT method intrinsically takes account of the interference processes taking place in a dynamical quantum mechanical system. Developed intensely in the last two decades, OCT utilizes time propagation operators. In more advanced application of this approach, a toolkit of time propagation operators to be stored and recalled as needed, is incorporated into the algorithm for the optimal control of a quantum systems. ${ }^{8}$

In complex molecules with too many atoms, any change in vibration of a bond instantly effects the vibration of the other bonds. Thus, selective excitation of one mode is instantly coupled to several dissipative mode of energy re-distribution. In such cases, OCT turns out to be the most appropriate method. It is not always feasible to generate the laser fields exactly as demanded by OCT unless issues of design like appropriate envelope of the pulse, lower fluence, etc. are included in the scheme.

In this work, we present control experiments carried out on a computer to obtain optimal fields to produce population transfer from an initial state to a given excited target state of the diatomic molecules, HF and $\mathrm{OH}$. We have used an iterative method ${ }^{26}$ for optimization described in $\$ 2$. In $\S 3$, we have described the control system. The results are discussed in $\S 4$ and finally, we conclude with comments on insights obtained in $\S 5$. 


\section{Theory}

The formalism of OCT is based on the choice of the optimal cost functional, $J[\varepsilon(t)]$, which depends on an optimal driving field. The cost functional, $J[\varepsilon(t)]$, containing mainly three terms, takes the following shape $^{26}$

$$
\begin{aligned}
& J[\varepsilon(t)]=\left|\left\langle\psi_{i}(T) \mid \phi_{f}(T)\right\rangle\right|^{2}-\beta_{0} \int_{0}^{T}[\varepsilon(t)]^{2} \mathrm{~d} t \\
& -2 \operatorname{Re}\left[\left\langle\psi_{i}(T) \mid \phi_{f}(T)\right\rangle \int_{0}^{T}\left\langle\chi_{f}(t)\left|\frac{\partial}{\partial t}+i \hat{H}\right| \psi_{i}(t)\right\rangle \mathrm{d} t\right] .
\end{aligned}
$$

The first term known as objective in (1) is chosen such that for the maximal value of the cost functional, the field propagated system states, $\left.\psi_{i}\right|_{(=T)}=\phi_{1}(T)$, at the terminal time $T$ (evolved from an initial state, $\left.\psi_{i}\right|_{(t=T)}=\phi_{i}(0)$, has maximum overlap with the desired product state $\phi_{\mathrm{f}}(T)$.

The second term known as penalty term in (1) is designed to include all the physical processes that are deemed undesirable during the controlled evolution of the molecule. It includes the restriction on the strength of the optical field $\varepsilon(t)$ which might be expressed as the fluence term. The factor $\beta_{0}$ is a positive weighing parameter to adjust the contribution of the radiation energy to the functional.

The last term involving the Schrödinger equation is the dynamical constraint that should be followed exactly during the time evolution of the system. The function $\chi_{f}(t)$ can be regarded as a Lagrange multiplier introduced to assure satisfaction of the Schrödinger equation.

The Hamiltonian operator, $\hat{H}$, within the dipole approximation is defined as

$$
\hat{H}=\hat{H}_{0}+V-\mu \varepsilon(t),
$$

where $\hat{H}_{0}$ is the kinetic energy operator, $\mu$ is the dipole moment and $V$ is the potential energy. The term $\left\langle\psi_{i}(t) \mid \phi_{f}(t)\right\rangle$ multiplying the first integral in (1) is introduced to decouple the boundary conditions for determination of $\psi_{i}(t)$ and $\chi_{f}(t)$.

Each of these terms depends explicitly or implicitly on the unknown driving field $\varepsilon(t)$ and the goal is to maximize $J[\varepsilon(t)]$ for the determination of $\varepsilon(t)$ by demanding $J[\varepsilon(t)] / \partial \varepsilon=0$. Setting to zero the first order variations of the cost functional with respect to $\varepsilon(t), \psi_{i}(t)$ and $\chi_{f}(t)$ leads to the following non-linear equations

$$
\begin{aligned}
& i \frac{\partial \psi_{i}(t)}{\partial t}=\hat{H} \psi_{i}(t), \psi_{i}(0)=\phi_{i}(0), \\
& i \frac{\partial \chi_{f}(t)}{\partial t}=\hat{H} \chi_{f}(t), \chi_{f}(T)=\phi_{f}(T), \\
& \beta_{0} \varepsilon(t)=-\operatorname{Im}\left\langle\psi_{i}(t) \mid \phi_{f}(t)\right\rangle\left(\left\langle\chi_{f}(t)|\mu| \psi_{i}(t)\right\rangle\right) .
\end{aligned}
$$

A time-dependent Gaussian factor, $g(t)$, is applied to the penalty function that ensures practically feasible profile of the laser pulse with a suitable envelope ${ }^{27}$

$$
\varepsilon(t)=-\frac{g(t)}{\beta_{0}} \operatorname{Im}\left\langle\psi_{i}(t) \mid \phi_{f}(t)\right\rangle\left(\left\langle\chi_{f}(t)|\mu| \psi_{i}(t)\right\rangle\right),
$$

where

$$
g(t)=\exp \left(-(t-T / 2)^{2} /(T / 4)^{2}\right) .
$$

Other choices are possible for $g(t)$. We have reported elsewhere calculations carried out using a sinusoidal shape. ${ }^{28}$

Equations (3) and (4) give the evolving initial and final state of the system in time and to obtain the desired field using (6) both are required at that point of time. The coupled differential (3) and (4) should be solved iteratively due to their non-linear nature. For this purpose, we have used an iterative method proposed by Zhu et $a l^{26}$ described below to obtain the desired field.

On a first reading it appears obvious that setting up the cost functional as in (1) will naturally have an optimal solution for the field for the pre-determined task of maximizing population in the final state. However, optimization in a function space is an extremely complex task. Ordinarily, a direct excitation using the fundamental frequency corresponding to the energy gap between the initial and final states would set up Rabi oscillations between the two states. We are demanding that precisely at a time equal to a predetermined value (say, 30,000 a.u.), the population of the final state must be maximum. There are several dynamical possibilities for this to happen. In other words, there are several optimal solutions in the space of functions describing the electric field. We ask for the one with reasonable fluence and an experimentally achievable pulse shape. While here we address a rather simple problem of vibrational excitation in a diatomic, more complex problems of re- 
active nature may be also formulated using OCT. As can be seen from the power spectra of the field obtained using OCT, the mechanism of excitation even in such simple cases involves other than the fundamental, small contributions from other frequencies.

\subsection{Iterative method}

By taking an initial guess field, $\varepsilon_{\text {initial }}$, the system target state, $\chi_{f}(T)=\phi_{f}(T)$, is propagated backward in time to obtain $\phi_{f}(T)$ and system initial state, $\psi_{i}=\phi_{i}(0)$, is propagated forward in time to obtain $\psi_{i}(t)$. Using these states, $\chi_{f}(t)$ and $\psi_{i}(t)$, the new field, $\varepsilon(t)$, is obtained using (6). The system final state, $\chi_{f}(T)-\phi_{f}(T)$, and the initial state, $\psi_{i}=\phi_{i}(0)$, are again propagated backward and forward using this field to obtain the new field. This process is repeated again iteratively to obtain an optimal value of the field. A discrete propagation method is used to evaluate the algorithm, which is employed for the solution of these nonlinear equations. To solve the Schrödinger equation, we have used the second order split-operator method. ${ }^{29,30} \mathrm{An}$ iterative process analogous to the one discussed above is set-up in order to maximize the cost functional, $J$.

\subsection{Split-operator method}

Numerical calculation of the evolution of a quantum mechanical system presents a tricky situation since the exponential of kinetic and potential energy operators have to be applied separately and the result put together. These two operators do not commute and the results obtained using one sequence of operation are generally different from those obtained using the reverse sequence. The split-operator method ${ }^{29,30}$ utilizes the splitting of the kinetic energy operator, $\hat{H}_{0}$ for better numerical accuracy. The propagator can be approximated using second order split-operator method for a small time interval $\Delta t$ as (Note that $V$ here includes the field term)

$$
e^{ \pm(i / \hbar) \hat{H} \Delta t}=e^{ \pm(i / 2 \hbar) \hat{H}_{0} \Delta t} e^{ \pm(i / \hbar) V \Delta t} e^{ \pm(i / 2 \hbar) \hat{H}_{0} \Delta t},
$$

where + sign indicates the backward propagation and - sign indicates the forward propagation of the system states respectively.

The kinetic energy, $\hat{H}_{0}$, part of the propagator, $e^{ \pm(i / 2 \hbar) \hat{H}_{0} \Delta t}$, is calculated using the Fast Fourier Transform (FFT) method. ${ }^{31,32}$ The operator, $e^{ \pm(i / \hbar) V \Delta t}$, is diagonal in the coordinate space representation and is calculated by simple multiplicative process.
Several other methods may be used for the optimization of cost functional. Results using a conjugate gradient method ${ }^{33}$ are reported elsewhere in this journal. ${ }^{34}$

\section{The control system}

We have chosen two diatomic systems for studying the vibrational excitations from an initial state to a target state in a Morse potential of the $\mathrm{HF}$ and $\mathrm{OH}$ molecule in the prescribed time interval $T$. Our objective is to control the population transfer from an initial state to a target state.

We solve the Schrödinger equation for the bound states of $\mathrm{HF}$ and $\mathrm{OH}$ molecule using Fourier Grid Hamiltonian method ${ }^{35}$ to obtain vibrational energies and eigenfunctions. The initial state of the system is prepared from these eigenfunctions.

The Morse potential model for the anharmonic interaction of the atoms is

$$
V(x)=D_{e}\left[1 \cdot 0-e^{-\beta\left(x-x_{e}\right)}\right]^{2},
$$

where for $\mathrm{HF}$ the parameters ${ }^{36}$ are $D_{e}=$ $0.2550073497, x_{e}=1.7329$, and $\beta=1.1741$ in a.u., and the dipole moment used is given by ${ }^{36}$

$$
\mu(x)=\mu_{0} x e^{-\sigma x^{4}},
$$

where, $\mu_{0}=0.4535$ and $\sigma=0.0064$ in a.u.

And the parameters for $\mathrm{O}-\mathrm{H}$ are $^{37} . D_{e}=0.1994$, $x_{e}=1.821$, and $\beta=1.189$ in a.u., and the dipole moment used is given by ${ }^{38}$

$$
\mu(x)=\mu_{0} x e^{-x / 0 \cdot 6},
$$

where, $\mu_{0}=3.088$ in a.u.

\section{Results and discussion}

Our goal is to see if arbitrary vibrational states can be prepared coherently using the iterative algorithm and the pulse shape manipulation discussed earlier. We selected a few cases arbitrarily for this purpose.

\subsection{Vibrational excitation of HF: $v=2 \rightarrow v=4$}

In the first case considered, the initial wave function, $\psi_{i}(0)$, is chosen to be the Morse 2nd excited state, and the target wave function is chosen to be the 

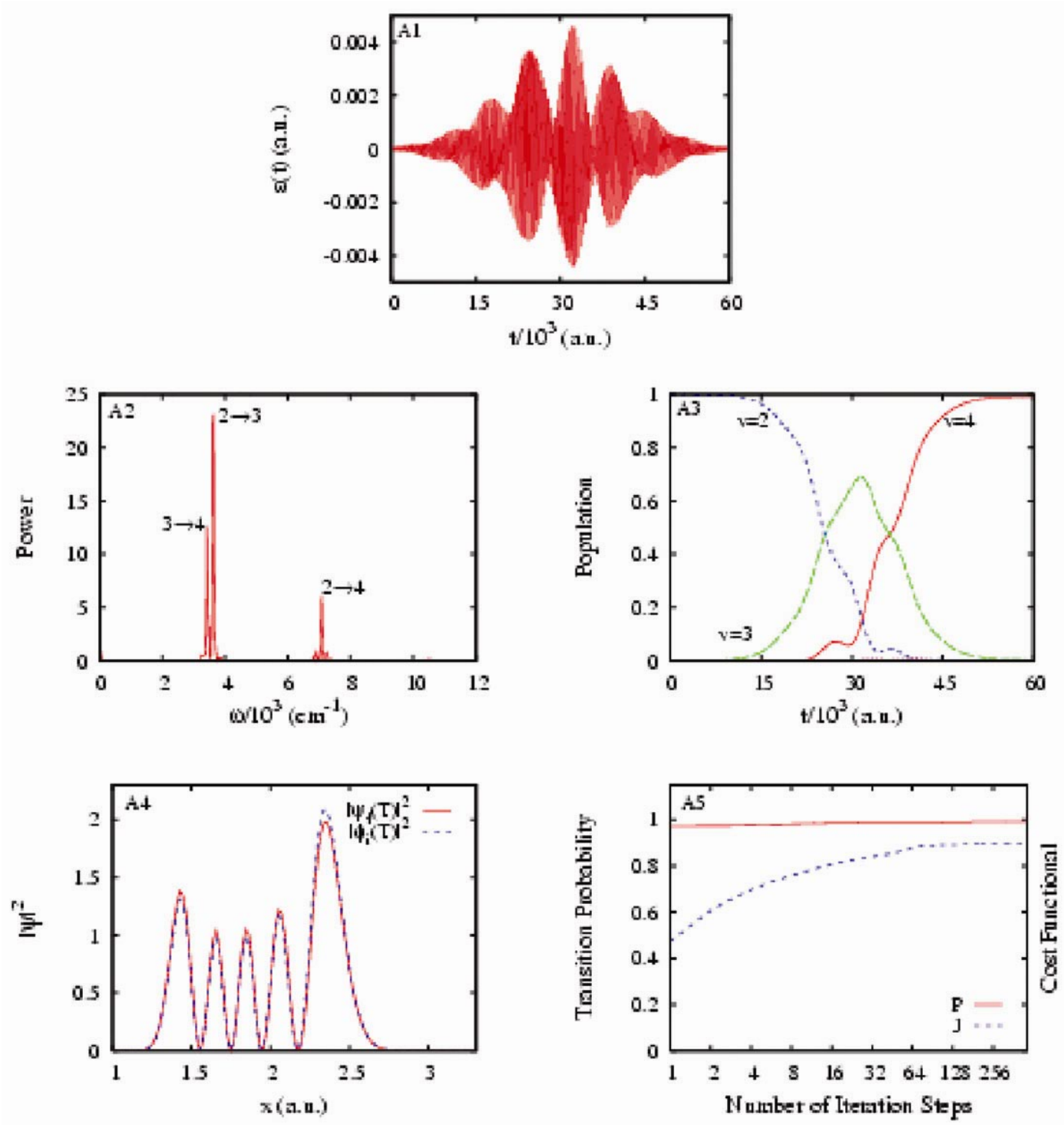

Figure 1. Results for $v=2 \rightarrow v=4$ of HF: Optimized field as a function of time (A1), power spectrum of the optimized field (A2), population analysis of relevant states as a function of time (A3), overlap of the field propagated state with target state at final time (A4), convergence of transition probability, $P$, and optimized cost functional, $J$, (A5) for a pulse 60,000 a.u.

Morse 4th excited state at 60000 a.u. in the ground electronic state of HF molecule. The initial guess for the field is taken as

$$
\varepsilon_{i}=0 \cdot 005\left[\sin \left(\omega_{23} t\right)+\sin \left(\omega_{34} t\right)\right],
$$

where $\omega_{23}$ and $\omega_{34}$ are the resonant transition frequencies between the 2nd and 3rd vibrational states and between the 3rd and 4th vibrational states of HF, respectively.
The optimized electric field corresponding to pulse duration of 60,000 a.u. is shown in plot A1 of figure 1 . The power spectrum of the optimized field is shown in plot A2 of figure 1 . It is clear from this figure that the field has major contribution from the frequencies corresponding to the following vibrational transitions $v=2 \rightarrow v=3, v=3 \rightarrow v=4$ and $v=2 \rightarrow v=4$ at 3623,3449 and $7072 \mathrm{~cm}^{-1}$ respectively. Thus the optimal solution under the given constraints is a mixture of several different transi- 

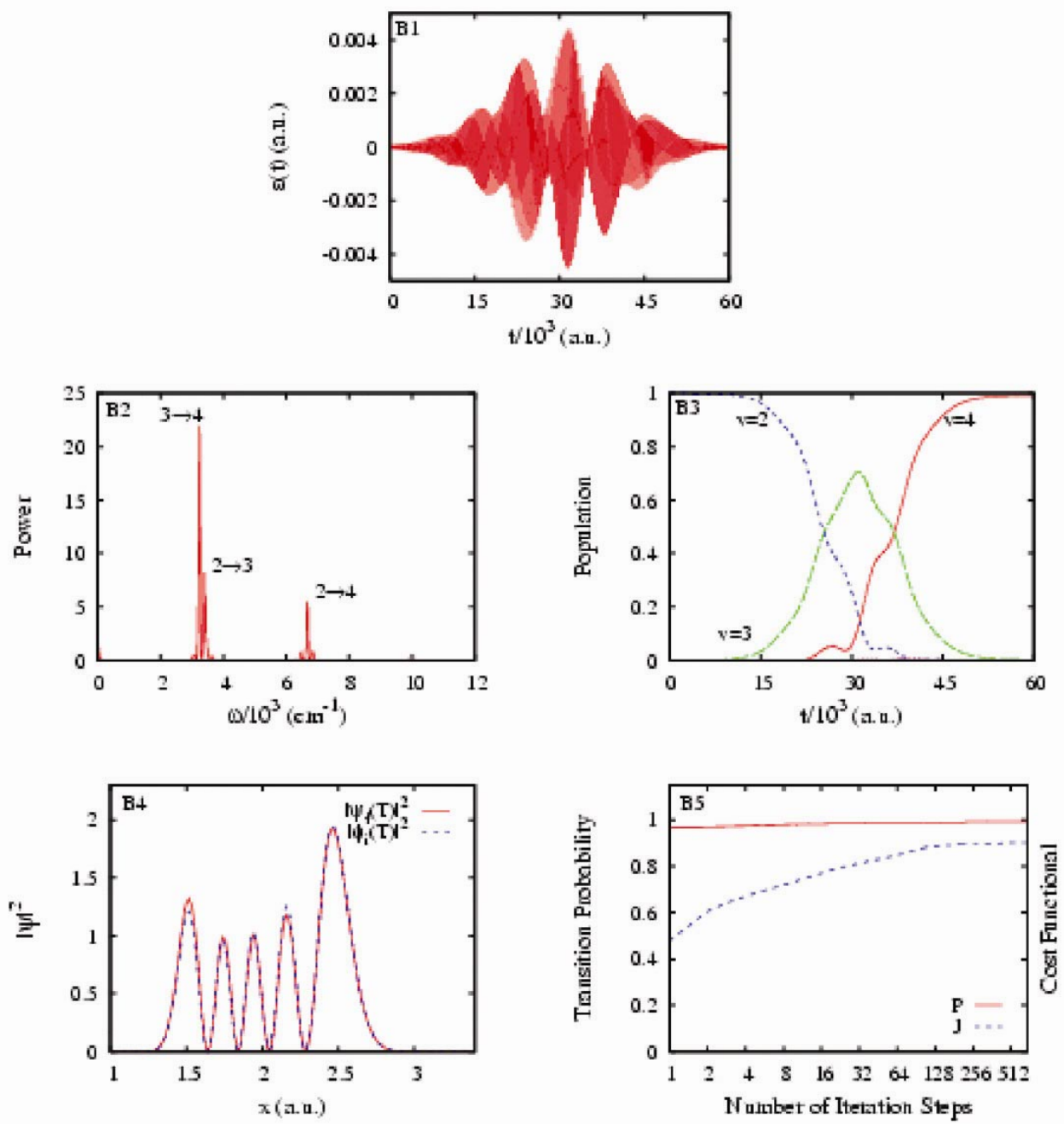

Figure 2. Results for $v=2 \rightarrow v=4$ of $\mathrm{OH}$ : Optimized field as a function of time (B1), power spectrum of the optimized field (B2), population analysis of relevant states as a function of time (B3), overlap of the field propagated state with target state at final time (B4), convergence of transition probability, $P$, and optimized cost functional, $J$, (B5) for a pulse 60,000 a.u.

tion frequencies rather than a single frequency corresponding to the energy gap between the initial and the final states.

The population of the field propagated initial state and the target state are plotted against time in plot A3 of figure 1 . There is a complete transfer of the population from the initial state to the desired target state. At first, we see an increase in the population of the intermediate $v=3$ state and then it decays to zero at the end of the pulse. The propagated initial state of the system at final time shows a large overlap with the target state as shown in plot A4 of figure 1 . These figures display the square of field propagated initial wave function and of the targeted wave function at final time.
Plot A5 of figure 1 shows the convergence behaviour of the objective functional as well as the optimized probability versus the number of iteration steps. The algorithm converges fast. The magnitude of the optimized objective functional is 0.89 corresponding to a $100 \%$ transfer of population to the final state.

\subsection{Vibrational excitation of $\mathrm{OH}: v=2 \rightarrow v=4$}

Next, we have obtained the optimal laser field for the vibrational transition from 2nd to 4th excited vibrational state in the ground electronic state of $\mathrm{OH}$ molecule. The initial guess of the field is same as described in (12) except the frequency now corre- 
Table 1. Comparison of results for $2 \rightarrow 4$ vibrational transition of $\mathrm{HF}$ and $\mathrm{OH}$ for a pulse duration of 60,000 a.u.

\begin{tabular}{lccccc}
\hline $\begin{array}{l}\text { Molecular } \\
\text { system }\end{array}$ & $\begin{array}{c}\text { Vibrational } \\
\text { transition }\end{array}$ & Energy & Iteration & $\begin{array}{c}\text { Transition } \\
\text { probability }(P)\end{array}$ & $\begin{array}{c}\text { Optimized cost } \\
\text { functional }(J)\end{array}$ \\
\hline $\mathrm{HF}$ & $2 \rightarrow 4$ & 7071 & 463 & $0 \cdot 99208$ & $0 \cdot 89831$ \\
$\mathrm{OH}$ & $2 \rightarrow 4$ & 6671 & 691 & 0.99246 & $0 \cdot 89983$ \\
\hline
\end{tabular}

sponds to the $\mathrm{OH}$ systems. The optimized electric field for this population transfer control is shown in plot B1 of figure 2 for 60,000 a.u. of time. The respective frequency distributions of the field plotted in B2 of figure 2 show major contributions from frequencies corresponding to the vibrational transitions $v=2 \rightarrow v=3, v=3 \rightarrow v=4$ and $v=2 \rightarrow v=4$ at 3425, 3246 and $6671 \mathrm{~cm}^{-1}$, respectively. It is interesting to note that while for $\mathrm{OH}$ the maximal contribution here comes from $v=3 \rightarrow v=4$ transition, whereas in case of HF, it was from $v=2 \rightarrow v=3$. Since we are dealing with the absorption of IR radiation caused by a change in dipole moment with nuclear motion, it is possible that this gradient in dipole moment is higher for $\mathrm{OH}$ as the energy of the system increases. This may explain the greater contribution from the $v=3 \rightarrow v=4$ frequency.

The population analysis of the initial and target states at final time is shown in plot B3 of figure 2 . The initial state corresponding to $v=2$ propagated by the optimal field roughly matches with the eigenstate corresponding to $v=4$ at final time as shown in plot B4 of figure 2. The transition probability and the final cost functional versus the number of iteration steps are shown in plot B5 of figure 2.

Comparing the results for the transition $v=2 \rightarrow$ $v=4$ in table 1 , it is seen that the transition probability is $\approx 100 \%$ for both the diatomic systems. There is not much difference in the numerical values of the cost functional as well as the transition probability for the two cases. Since the field peak magnitudes and envelopes are nearly similar, the fluence terms are of comparable magnitudes. Interestingly, the convergence in case of $\mathrm{OH}$ turned out to be much slower than in the case of HF.

\section{Conclusion}

The iterative method described in §2 was applied for the control of vibrational excitations in two diatomic quantum systems. We obtained reliable desired laser field for the deterministic control problems. A Gaussian envelope factor is introduced into the field equation for meaningful practical realizations of the field into the laboratory. We have also explored the possibility of the field shape using a sinusoidal factor reported elsewhere ${ }^{28}$. There is a slight disadvantage of the Gaussian shape ${ }^{3}$ of the field envelope, it does not go strictly to zero before and after the pulse. But the overall shape of the field corresponds more closely to experimentally realizable possibilities. Fields were optimized for the vibrational transition from $v=2 \rightarrow$ $v=4$ described above for $\mathrm{HF}$ and $\mathrm{OH}$. The peak amplitude of the field is within reasonable and experimentally realizable bounds. In our further work, we are exploring the role of environmental noise on the control scenario.

\section{Acknowledgments}

Authors acknowledge support from the Department of Science and Technology, Govt. of India, New Delhi.

\section{References}

1. Rice S and Zhao M 2000 Optical control of molecular dynamics (John Wiley \& Sons: NY)

2. (a) Shapiro M and Brumer P 2003 Principles of the quantum control of molecular processes (John Wiley \& Sons); (b) Goswami D 2003 Phys. Rep. 374 385

3. Balint-Kurti G G, Zou S and Brown A Adv. Chem. Phys. (in press)

4. Brumer P and Shapiro M March 1995 Scientific Am. 55

5. Tannor D J and Rice S A 1985 J. Chem. Phys. 83 5013

6. Tannor D J, Kosloff R and Rice S A 1986 J. Chem. Phys. 855805

7. Tannor D J and Rice S A 1988 Adv. Chem. Phys. 70 441

8. Yip F, Mazziotti D and Rabitz H 2003 J. Chem. Phys. 1188168

9. (a) Park S M, Lu S-P and Gordon R J 1991 J. Chem. Phys. 94 8622; (b) Lu S-P, Park S M, Xie Y and Gordon R L 1992 J. Chem. Phys. 96 6613; (c) Zhu L, Kleiman V, Li X, Lu S-P, Trentelman K and Gordon R J 1995 Science 27077

10. Levy I, Shapiro M and Brumer P 1990 J. Chem. Phys. 932493 
11. Brown A and Balint-Kurti G G 2000 J. Chem. Phys. 1131879

12. Regan P M, Ascenzi D, Brown A, Balint-Kurti G G and Orr-Ewing A J 2000 J. Chem. Phys. 11210259

13. Sinha A, Hsiao M C and Crim F F $1990 \mathrm{~J}$. Chem. Phys. 926333

14. Zare R N 1998 Science 2791875

15. Shapiro M and Brumer P 1993 J. Chem. Phys. 98201

16. Dantus M, Rosker M J and Zewail A H $1988 \mathrm{~J}$. Chem. Phys. 896128

17. Rosker M J, Dantus M and Zewail A H 1988 Science 2541200

18. Pastirk I, Brown E J, Zhang Q and Dantus M $1998 \mathrm{~J}$. Chem. Phys. 1084375

19. Gross P, Singh H, Rabitz H, Measer K and Huang G M 1993 Phys. Rev. A47 4593

20. Meier C and Heitz M-C 2005 J. Chem. Phys. 23 044504

21. Pearson B J, White L J, Weinacht T C and Bucksbaum P H 2001 Phys. Rev. A63 063412

22. Zeidler D, Frey S, Kompa K L and Motzkus M 2001 Phys. Rev. A64 023420

23. Judson R S and Rabitz H 1992 Phys. Rev. Lett. 68 1500

24. Amstrup B, Toth G J, Szabo G and Lörincz A $1995 \mathrm{~J}$. Phys. Chem. 995206
25. Amstrup B, Doll J D, Sauerbrey R A, Szabo G and Lörincz A 1993 Phys. Rev. A48 3830

26. Zhu W, Botina J and Rabitz H 1998 J. Chem. Phys. 1081953

27. Sundermann K and Vivie-Riedle R de 1999 J. Chem. Phys. 1101896

28. Kumar P, Sharma $S$ and Singh $H$ (communicated)

29. Feit M D and Fleck Jr J A 1983 J. Chem. Phys. 78 301

30. Feit M D and Fleck Jr J A 1984 J. Chem. Phys. 80 2578

31. Press W H, Flannery B P, Teukolsky S A and Vetterling 1993 Numerical recipes (Cambridge: Cambridge University)

32. Cooley J W and Tukey J W 1965 Math. Comput. 19 297

33. Balint-Kurti G G, Manby F R, Ren Q, Artamonov M, Ho Tak-San and Rabitz H 2005 J. Chem. Phys. 122 084110

34. Sharma S, Sharma P and Singh H 2007 J. Chem. Sci. (this issue)

35. Marston C C and Balint-Kurti G G 1989 J. Chem. Phys. 913571

36. Stine J R and Noid D W 1979 Opt. Commun. 31161

37. Paramonov G K 1993 Chem. Phys. 177169

38. Lawton R T and Child M S 1980 Mol. Phys. 40773 